\title{
Correction to: Efficient tunable plasmonic mode converters infiltrated with nematic liquid crystal layers
}

\author{
Randa H. Kabeel ${ }^{1} \cdot$ Nihal F. F. Areed $^{1}$ - Mohamed Farhat O. Hameed ${ }^{2,3,4}$. \\ Salah S. A. Obayya ${ }^{1,2}$ \\ Published online: 6 August 2021 \\ @ The Author(s), under exclusive licence to Springer Science+Business Media, LLC, part of Springer Nature 2021
}

\section{Correction to: Optical and Quantum Electronics (2021) 53:436 https://doi.org/10.1007/s11082-021-03086-5}

In the original publication of the article, the article title was published with an error and the author name Salah S. A. Obayya was incorrectly written as Salah S. A. Salah. These have been corrected with this Correction.

The original article has been corrected.

Publisher's Note Springer Nature remains neutral with regard to jurisdictional claims in published maps and institutional affiliations.

The original article can be found online at https://doi.org/10.1007/s11082-021-03086-5.

Mohamed Farhat O. Hameed mfarahat@zewailcity.edu.eg

$\bowtie$ Salah S. A. Obayya sobayya@zewailcity.edu.eg

1 Department of Electronics and Communications Engineering, Faculty of Engineering, University of Mansoura, Mansoura 35516, Egypt

2 Centre for Photonics and Smart Materials, Zewail City of Science and Technology, October Gardens, 6th of October City, Giza 12578, Egypt

3 Nanotechnology and Nanoelectronics Engineering Program, Zewail City of Science and Technology, October Gardens, 6th of October City, Giza 12578, Egypt

4 Mathematics and Engineering Physics Department, Faculty of Engineering, University of Mansoura, Mansoura 35516, Egypt 